\title{
Variational proof of the existence of eigenvalues for star graphs
}

\author{
Konstantin Pankrashkin \\ Dedicated to Pavel Exner on the occasion of his 70th birthday
}

\begin{abstract}
We provide a purely variational proof of the existence of eigenvalues below the bottom of the essential spectrum for the Schrödinger operator with an attractive $\delta$-potential supported by a star graph, i.e. by a finite union of rays emanating from the same point. In contrast to the previous works, the construction is valid without any additional assumption on the number or the relative position of the rays. The approach is used to obtain an upper bound for the lowest eigenvalue.
\end{abstract}

\section{Introduction}

The mathematically rigorous study of multidimensional Schrödinger operators with potentials supported by hypersurfaces was initiated in 1994 by Brasche, Exner, Kuperin and Šeba [2]. The two-dimensional Hamiltonians with interactions supported by curves have become a prominent class of solvable models of quantum mechanics $\mathbf{8}$ and are usually referred to as leaky quantum graphs. A summary of various questions and results in the spectral theory of such operators can be found in the review by Exner [7], and for the most recent developments we refer to the papers $[\mathbf{1}, \mathbf{5}, \mathbf{1 0}, \mathbf{1 4}, \mathbf{1 5}, \mathbf{1 7}, \mathbf{1 8}$ and to Chapter 10 in the recent monograph by Exner and Kovařík [1].

In the present contribution, we are interested in some properties of Schrödinger operators with $\delta$-interactions supported by the so-called star graphs. By a star graph $\Gamma$ we mean a subset of $\mathbb{R}^{2}$ obtained as the union of finitely many rays emanating from the origin. If $(r, \theta)$ is the standard polar coordinate system, then $\Gamma$ is naturally identified with a family $\left(\theta_{1}, \ldots, \theta_{N}\right)$ in which $0 \leq \theta_{1}<\cdots<\theta_{N}<$ $2 \pi$ by $\Gamma:=\bigcup_{j=1}^{N}\left\{(r, \theta): \theta=\theta_{j}, r \geq 0\right\}$. The associated Schrödinger operator $H_{\Gamma, \alpha}=-\Delta-\alpha \delta_{\Gamma}$, where $\delta_{\Gamma}$ is the Dirac $\delta$-distribution supported by $\Gamma$ and $\alpha>0$ is a coupling constant, is defined as the unique self-adjoint operator in $L^{2}\left(\mathbb{R}^{2}\right)$ associated with the closed lower semibounded quadratic form

$$
Q_{\Gamma, \alpha}(u)=\iint_{\mathbb{R}^{2}}|\nabla u|^{2} d x-\alpha \int_{\Gamma}|u|^{2} d s, \quad u \in H^{1}\left(\mathbb{R}^{2}\right) .
$$

where $d s$ is the one-dimensional Hausdorff measure, cf. 2]. Such configurations appear naturally as a mathematical model for a junction of quantum wires, and they were first analyzed by Exner and Němcová 12, 13. The basic spectral properties of the operator are well known: the essential spectrum coincides with the semi-axis $\left[-\alpha^{2} / 4,+\infty\right)$, and the discrete spectrum is non-empty except in the degenerate cases when $\Gamma$ is a single ray $(N=1)$ or a straight line $\left(N=2\right.$ and $\left.\left|\theta_{1}-\theta_{2}\right|=\pi\right)$. 
Despite the simple geometrical picture, the only available proof of the existence of eigenvalues is based on a rather involved analysis of integral operators carried out by Exner and Ichinose 9. On the other hand, by the min-max principle, the non-emptiness of the discrete spectrum would follow from the existence of a trial function $v \in H^{1}\left(\mathbb{R}^{2}\right)$ satisfying the strict inequality

$$
Q_{\Gamma, \alpha}(v)<-\frac{\alpha^{2}}{4}\|v\|_{L^{2}\left(\mathbb{R}^{2}\right)}^{2}
$$

Surprisingly, the construction of such a function appeared to be a difficult task. The construction of Exner and Němcová $1 \mathbf{1 3}$. works only if there is a pair of rays with $\left|\theta_{j}-\theta_{k}\right| \bmod 2 \pi<0.092$. Brown, Eastham and Wood [3, 4, 6 managed to find a trial function for all possible configurations with $N \geq 3$ as well as for the configurations with $N=2$ and $\left|\theta_{1}-\theta_{2}\right|<0.9271$. In the present note we show how to construct such a function for all possible cases (Theorem 1), and our approach uses a likeliness between the star graphs and a spectral problem of the surface superconductivity with a similar geometry discussed by Lu and Pan 19 and Helffer and Morame 16. We remark again that Theorem 1 itself does not provide any new spectral information, but suggests a new method to show the presence of a non-empty discrete spectrum as an alternative to the analytical proof by Exner and Ichinose [9. On the other hand, the presence of explicitly given trial functions allows one to obtain a universal upper bound for the lowest eigenvalue (Theorem 2), which is a new result.

\section{Construction of a trial function}

By the min-max principle, it is sufficient to consider the case $N=2$ (a broken line), then, up to isometries, all possible configurations can be described by a single parameter $\theta \in(0, \pi / 2)$ through $\Gamma=\overline{\Gamma_{+} \cup \Gamma_{-}}$with $\Gamma_{ \pm}:=\{(t, \pm t \tan \theta): t>0\}$, and the associated operator $H_{\Gamma, \alpha}$ will be denoted by $H(\theta, \alpha)$.

We remark first that in order to show that the discrete spectrum is non-empty it is sufficient to consider the problem in the half-plane $\mathbb{R} \times \mathbb{R}_{+}$, i.e. to find a function $u \in H^{1}\left(\mathbb{R} \times \mathbb{R}_{+}\right)$satisfying

$$
\iint_{\mathbb{R} \times \mathbb{R}_{+}}|\nabla u|^{2} d x-\alpha \int_{\Gamma_{+}}|u|^{2} d x<-\frac{\alpha^{2}}{4}\|u\|_{L^{2}\left(\mathbb{R} \times \mathbb{R}_{+}\right)}^{2},
$$

as its extension $v$ to the whole of $\mathbb{R}^{2}$ by parity automatically satisfies (11). For subsequent constructions, it is handy to perform an additional rotation to put the support of the interaction onto the positive semi-axis of ordinates. In other words, we will work with the domain $\Omega:=\left\{\left(x_{1}, x_{2}\right): x_{1}<x_{2} \tan \theta\right\}$ and the quadratic form

$$
Q(u)=\iint_{\Omega}|\nabla u|^{2} d x-\alpha \int_{\mathbb{R}_{+}}\left|u\left(0, x_{2}\right)\right|^{2} d x_{2}, \quad u \in H^{1}(\Omega) .
$$

THEOREM 1. Pick any $\rho \in\left(0, \cot ^{2} \theta\right)$ and any Lipschitz function $\chi: \mathbb{R} \rightarrow[0,1]$ with $\chi(t)=1$ for $|t| \leq 1$ and $\chi(t)=0$ for $|t| \geq 2$, then for sufficiently large $n>0$ the function $u$ defined by

$$
u\left(x_{1}, x_{2}\right)=e^{-\alpha\left|x_{1}\right| / 2}\left(\frac{2}{\alpha} \mathbf{1}_{\mathbb{R}_{+}}\left(x_{2}\right)-\frac{1}{\alpha} e^{-\alpha\left|x_{2}\right| \tan \theta} \operatorname{sgn} x_{2}\right)^{\rho} \chi\left(\frac{x_{2}}{n}\right)
$$

satisfies the strict inequality $Q(u)<-\frac{\alpha^{2}}{4}\|u\|_{L^{2}(\Omega)}^{2}$.

Proof. For futher use, denote

$$
F(t):=\int_{-\infty}^{t} e^{-\alpha\left|x_{1}\right|} d x_{1}=\frac{2}{\alpha} \mathbf{1}_{\mathbb{R}_{+}}(t)-\frac{1}{\alpha} e^{-\alpha|t|} \operatorname{sgn} t .
$$


For the functions $u$ of the form $u\left(x_{1}, x_{2}\right)=e^{-\alpha\left|x_{1}\right| / 2} g\left(x_{2}\right)$ with real-valued $g$ we have

$$
\|u\|_{L^{2}(\Omega)}^{2}=\int_{\mathbb{R}} \int_{-\infty}^{x_{2} \tan \theta} e^{-\alpha\left|x_{1}\right|} g\left(x_{2}\right)^{2} d x_{1} d x_{2}=\int_{\mathbb{R}} g\left(x_{2}\right)^{2} F\left(x_{2} \tan \theta\right) d x_{2} .
$$

Furthermore,

$$
\begin{aligned}
Q(u)=\frac{\alpha^{2}}{4} \int_{\mathbb{R}} g\left(x_{2}\right)^{2} \int_{-\infty}^{x_{2} \tan \theta} e^{-\alpha\left|x_{1}\right|} d x_{1} d x_{2} & \\
& \quad+\int_{\mathbb{R}} g^{\prime}\left(x_{2}\right)^{2} \int_{-\infty}^{x_{2} \tan \theta} e^{-\alpha\left|x_{1}\right|} d x_{1} d x_{2}-\alpha \int_{\mathbb{R}_{+}} g\left(x_{2}\right)^{2} d x_{2} .
\end{aligned}
$$

Due to

$$
\begin{gathered}
\frac{\alpha^{2}}{4} \int_{-\infty}^{x_{2} \tan \theta} e^{-\alpha\left|x_{1}\right|} d x_{1}=\frac{\alpha^{2}}{4} F\left(x_{2} \tan \theta\right)=-\frac{\alpha^{2}}{4} F\left(x_{2} \tan \theta\right)+\frac{\alpha^{2}}{2} F\left(x_{2} \tan \theta\right) \\
=-\frac{\alpha^{2}}{4} F\left(x_{2} \tan \theta\right)+\alpha \mathbf{1}_{\mathbb{R}_{+}}\left(x_{2}\right)-\frac{\alpha}{2} e^{-\alpha\left|x_{2}\right| \tan \theta} \operatorname{sgn} x_{2}
\end{gathered}
$$

we have

(4) $Q(u)=-\frac{\alpha^{2}}{4} \int_{\mathbb{R}} g\left(x_{2}\right)^{2} F\left(x_{2} \tan \theta\right) d x_{2}+\int_{\mathbb{R}} g^{\prime}\left(x_{2}\right)^{2} F\left(x_{2} \tan \theta\right) d x_{2}$

$$
-\frac{\alpha}{2} \int_{\mathbb{R}} g\left(x_{2}\right)^{2} e^{-\alpha\left|x_{2}\right| \tan \theta} \operatorname{sgn} x_{2} d x_{2} .
$$

Using the integration by parts we obtain

$$
\begin{gathered}
\int_{\mathbb{R}} g\left(x_{2}\right)^{2} e^{-\alpha\left|x_{2}\right| \tan \theta} \operatorname{sgn} x_{2} d x_{2}=\frac{2}{\alpha} \cot \theta \int_{\mathbb{R}} g\left(x_{2}\right) g^{\prime}\left(x_{2}\right) e^{-\alpha\left|x_{2}\right| \tan \theta} d x_{2} \\
=\frac{2}{\alpha} \cot \theta \int_{\mathbb{R}} g\left(x_{2}\right) g^{\prime}\left(x_{2}\right) F^{\prime}\left(x_{2} \tan \theta\right) d x_{2},
\end{gathered}
$$

and the substitution of (3) and (5) into (4) gives the representation

$$
\begin{aligned}
& Q(u)=-\frac{\alpha^{2}}{4}\|u\|_{L^{2}(\Omega)}^{2}+R(g), \\
& R(g):=\int_{\mathbb{R}} g^{\prime}\left(x_{2}\right)\left(g^{\prime}\left(x_{2}\right) F\left(x_{2} \tan \theta\right)-g\left(x_{2}\right) F^{\prime}\left(x_{2} \tan \theta\right) \cot \theta\right) d x_{2} .
\end{aligned}
$$

Hence, we need to find a function $g$ with $R(g)<0$.

Pick $\rho \in\left(0, \cot ^{2} \theta\right)$ and introduce a function $g_{\rho}$ by $g_{\rho}\left(x_{2}\right)=F\left(x_{2} \tan \theta\right)^{\rho}$, then

$$
R\left(g_{\rho}\right)=\rho \tan ^{2} \theta\left(\rho-\cot ^{2} \theta\right) \int_{\mathbb{R}} e^{-2 \alpha\left|x_{2}\right| \tan \theta} F\left(x_{2} \tan \theta\right)^{2 \rho-1} d x_{2}<0 .
$$

Remark that the integral is finite, but the function $g_{\rho}$ has a non-zero finite limit at $+\infty$, and the associated function $u$ does not belong to $H^{1}(\Omega)$ due to (3).

Choose a Lipschitz function $\chi: \mathbb{R} \rightarrow[0,1]$ with $\chi(t)=1$ for $|t| \leq 1$ and $\chi(t)=0$ for $|t| \geq 2$, and for $n>0$ denote $h_{n}:=g_{\rho} \chi(\cdot / n)$. By construction, the associated functions $u_{n}$ given by

$$
u_{n}\left(x_{1}, x_{2}\right)=e^{-\alpha\left|x_{1}\right| / 2} h_{n}\left(x_{2}\right),
$$


belong to $H^{1}(\Omega)$ and coincide with (2). In addition,

$$
\begin{gathered}
R\left(h_{n}\right)-R\left(g_{\rho}\right) \\
=\int_{\mathbb{R}}\left(\chi\left(\frac{x_{2}}{n}\right)^{2}-1\right) g_{\rho}^{\prime}\left(x_{2}\right)\left(g_{\rho}^{\prime}\left(x_{2}\right) F\left(x_{2} \tan \theta\right)-g_{\rho}\left(x_{2}\right) F^{\prime}\left(x_{2} \tan \theta\right) \cot \theta\right) d x_{2} \\
+\frac{1}{n} \int_{\mathbb{R}} \chi\left(\frac{x_{2}}{n}\right) \chi^{\prime}\left(\frac{x_{2}}{n}\right)\left(2 g_{\rho}\left(x_{2}\right) g_{\rho}^{\prime}\left(x_{2}\right) F\left(x_{2} \tan \theta\right)-g_{\rho}\left(x_{2}\right)^{2} F^{\prime}\left(x_{2} \tan \theta\right) \cot \theta\right) d x_{2} \\
+\frac{1}{n^{2}} \int_{\mathbb{R}} \chi^{\prime}\left(\frac{x_{2}}{n}\right)^{2} g_{\rho}\left(x_{2}\right)^{2} F\left(x_{2} \tan \theta\right) d x_{2}=: I_{1}+I_{2}+I_{3} .
\end{gathered}
$$

Due to the finiteness of $R\left(g_{\rho}\right)$, for large $n$ we have

$$
\begin{aligned}
\left|I_{1}\right| \leq & \int_{\mathbb{R} \backslash(-n, n)}\left|g_{\rho}^{\prime}\left(x_{2}\right)\left(g_{\rho}^{\prime}\left(x_{2}\right) F\left(x_{2} \tan \theta\right)-g_{\rho}\left(x_{2}\right) F^{\prime}\left(x_{2} \tan \theta\right) \cot \theta\right)\right| d x_{2} \\
& =\rho \tan ^{2} \theta \cdot\left|\rho-\cot ^{2} \theta\right| \int_{\mathbb{R} \backslash(-n, n)} e^{-2 \alpha\left|x_{2}\right| \tan \theta} F\left(x_{2} \tan \theta\right)^{2 \rho-1} d x_{2}=o(1) .
\end{aligned}
$$

Furthermore,

$$
\begin{aligned}
\left|I_{2}\right| & =\left|\frac{1}{n} \int_{\mathbb{R}}(2 \rho \tan \theta-\cot \theta) \chi\left(\frac{x_{2}}{n}\right) \chi^{\prime}\left(\frac{x_{2}}{n}\right) e^{-\alpha\left|x_{2}\right| \tan \theta} F\left(x_{2} \tan \theta\right)^{2 \rho} d x_{2}\right| \\
& \leq \frac{\left|2 \rho-\cot ^{2} \theta\right| \cdot \tan \theta \cdot\left\|\chi^{\prime}\right\|_{\infty}}{n} \int_{\mathbb{R}} e^{-\alpha\left|x_{2}\right| \tan \theta} F\left(x_{2} \tan \theta\right)^{2 \rho} d x_{2}=\mathcal{O}\left(\frac{1}{n}\right)
\end{aligned}
$$

due to the convergence of the integral. Finally, as the integrand is bounded, we have

$$
\left|I_{3}\right| \leq \frac{1}{n^{2}}\left(\int_{-2 n}^{-n}+\int_{n}^{2 n}\left\|\chi^{\prime}\right\|_{\infty}^{2} g_{\rho}\left(x_{2}\right)^{2} F\left(x_{2} \tan \theta\right) d x_{2}\right)=\frac{1}{n^{2}} \cdot \mathcal{O}(n)=\mathcal{O}\left(\frac{1}{n}\right),
$$

and we arrive at $R\left(h_{n}\right)=R\left(g_{\rho}\right)+o(1)$ as $n$ tends to $+\infty$. As $R\left(g_{\rho}\right)<0$, we have $R\left(h_{n}\right)<0$ for large $n$, which shows that the functions (6) have the sought property.

\section{Upper bound for the lowest eigenvalue}

We remark first that various estimates for the lowest eigenvalue $\lambda(\theta, \alpha)$ of $H(\theta, \alpha)$ were obtained in earlier works. In particular, Duchêne and Raymond $\mathbf{5}$ showed that

$$
\lambda(\theta, \alpha)=-\alpha^{2}\left[1-c_{1} \theta^{2 / 3}+\mathcal{O}(\theta)\right], \quad \theta \rightarrow 0+,
$$

and Exner and Kondej [10] proved that

$$
\lambda(\theta, \alpha)=-\alpha^{2}\left[\frac{1}{4}+c_{2}\left(\frac{\pi}{2}-\theta\right)^{4}+o\left(\left(\frac{\pi}{2}-\theta\right)^{4}\right)\right], \quad \theta \rightarrow \frac{\pi}{2}-,
$$

where $c_{1}$ and $c_{2}$ are some explicit positive constants.

Recall that by the min-max principle there holds $\lambda(\theta, \alpha) \leq Q(v) /\|v\|_{L^{2}(\Omega)}^{2}$ for any non-zero $v \in H^{1}(\Omega)$. We would like to use the trial functions $u$ from Theorem 1 to obtain an explicit upper estimate for the eigenvalue valid for all values of $\theta$. As the limit $\lim _{n \rightarrow+\infty} Q(u) /\|u\|_{L^{2}(\Omega)}^{2}=-\alpha^{2} / 4$ coincides with the bottom of the essential spectrum, we cannot hope for the best possible result. Nevertheless, the estimate and the method can be of some interest as, to our best knowledge, no analogous bound has been available so far. 
Theorem 2. For any $\theta \in\left(0, \frac{\pi}{2}\right)$ there holds $\lambda(\theta, \alpha) \leq-\alpha^{2}\left(\frac{1}{4}+\Lambda(\theta)\right)$, where

(9) $\Lambda(\theta):=\frac{3 \cos ^{6} \theta\left(2^{2 \cos ^{2} \theta}-1\right)^{2}}{2\left(1+2 \cos ^{2} \theta\right)^{3}\left(108+180 \cos ^{2} \theta-132 \cos ^{4} \theta+45 \cos ^{6} \theta-5 \cos ^{8} \theta\right)}$

is strictly positive.

A comparison with (7) and (8) shows that the upper estimate is away of an optimal one. For $\theta$ close to 0 our estimate gives $\lambda(\theta, \alpha) \leq-99 \alpha^{2} / 392+\mathcal{O}(\theta)$ which is very weak when compared with the true behavior given by (77). At $\theta=\pi / 2$, the value of $\Lambda(\theta)$ vanishes at the tenth order, which is also very far from the true fourth order given in (8). Our bound resulted from various experiments with the parameters and used a number of very rough inequalities, and the interested reader should feel free to improve the estimate using an alternative choice of parameters.

Proof. The result is based on a more accurate estimate of the quantities appearing in the proof of Theorem 1 for an explicit choice of the function $\chi$ and of the parameter $\rho$. Namely, we set

$$
\chi(t):= \begin{cases}1, & |t| \leq 1 \\ 2-|t|, & |t| \in(1,2), \quad \rho:=\cos ^{2} \theta \\ 0, & |t| \geq 2\end{cases}
$$

then $\left\|\chi^{\prime}\right\|_{\infty}=1$. We have

$$
\begin{aligned}
R\left(g_{\rho}\right) & =\frac{\rho \tan ^{2} \theta\left(\rho-\cot ^{2} \theta\right)}{\alpha^{2 \rho-1}} \\
& \times\left(\int_{-\infty}^{0} e^{(2 \rho+1) x_{2} \tan \theta} d x_{2}+\int_{0}^{+\infty} e^{-2 \alpha x_{2} \tan \theta}\left(2-e^{-\alpha x_{2} \tan \theta}\right)^{2 \rho-1} d x_{2}\right) .
\end{aligned}
$$

We calculate

$$
\int_{-\infty}^{0} e^{(2 \rho+1) x_{2} \tan \theta} d x_{2}=\frac{1}{(2 \rho+1) \alpha \tan \theta}
$$

and, using the change of variables $s=e^{-\alpha x_{2} \tan \theta}$,

$$
\begin{aligned}
& \int_{0}^{+\infty} e^{-2 \alpha x_{2} \tan \theta}\left(2-e^{-\alpha x_{2} \tan \theta}\right)^{2 \rho-1} d x_{2}=\frac{1}{\alpha \tan \theta} \int_{0}^{1} s(2-s)^{2 \rho-1} d s \\
& =\frac{1}{\alpha \tan \theta} \int_{0}^{1}\left(2(2-s)^{2 \rho-1}-(2-s)^{2 \rho}\right) d s=\frac{1}{\alpha \tan \theta}\left(\frac{2^{2 \rho}-1}{\rho}-\frac{2^{2 \rho+1}-1}{2 \rho+1}\right)
\end{aligned}
$$

which gives

$$
R\left(g_{\rho}\right)=\frac{1}{\alpha^{2 \rho}} \frac{\tan \theta\left(\rho-\cot ^{2} \theta\right)\left(2^{2 \rho}-1\right)}{2 \rho+1}=-\frac{\cos ^{3} \theta\left(2^{2 \cos ^{2} \theta}-1\right)}{\sin \theta\left(1+2 \cos ^{2} \theta\right)} .
$$

In what follows we will use the following estimates valid for $s \in[0,1]$ due to the convexity argument:

$$
\frac{1}{1+2 s} \leq 1-\frac{2}{3} s, \quad \frac{1}{(1+2 s)^{2}} \leq 1-\frac{8}{9} s, \quad 2^{2 s} \leq 1+3 s .
$$


We estimate

$$
\begin{aligned}
\left|I_{1}\right| \leq & \frac{\cos ^{4} \theta}{\alpha^{2 \rho-1}}\left(\int_{-\infty}^{-n} e^{(2 \rho+1) \alpha x_{2} \tan \theta} d x_{2}\right. \\
& \left.\quad+\int_{n}^{+\infty} e^{-2 \alpha x_{2} \tan \theta}\left(2-e^{-\alpha x_{2} \tan \theta}\right)^{2 \rho-1} d x_{2}\right) \\
\leq & \frac{\cos ^{4} \theta}{\alpha^{2 \rho-1}}\left(\int_{-\infty}^{-n} e^{(2 \rho+1) \alpha x_{2} \tan \theta} d x_{2}+2^{2 \rho} \int_{n}^{+\infty} e^{-2 \alpha x_{2} \tan \theta} d x_{2}\right) \\
= & \frac{\cos ^{4} \theta}{\alpha^{2 \rho-1}}\left(\frac{1}{(2 \rho+1) \alpha \tan \theta} e^{-(2 \rho+1) \alpha n \tan \theta}+\frac{2^{2 \rho}}{2 \alpha \tan \theta} e^{-2 \alpha n \tan \theta}\right) \\
\leq & \frac{\cos ^{4} \theta}{\alpha^{2 \rho-1}}\left(\frac{1}{((2 \rho+1) \alpha \tan \theta)^{2} n}+\frac{2^{2 \rho}}{(2 \alpha \tan \theta)^{2} n}\right) \\
\leq & \frac{1}{\alpha^{2 \rho+1}} \cdot \frac{\cos ^{6} \theta}{\sin ^{2} \theta}\left(\frac{1}{(2 \rho+1)^{2}}+\frac{1}{4} \cdot 2^{2 \rho}\right) \cdot \frac{1}{n} \\
\leq & \frac{1}{\alpha^{2 \rho+1}} \cdot \frac{\cos ^{6} \theta}{\sin ^{2} \theta}\left(1-\frac{8}{9} \rho+\frac{1}{4}(1+3 \rho)\right) \cdot \frac{1}{n} \\
= & \frac{1}{\alpha^{2 \rho+1}} \cdot \frac{45 \cos ^{6} \theta-5 \cos ^{8} \theta}{36 \sin ^{2} \theta} \cdot \frac{1}{n}
\end{aligned}
$$

and

$$
\begin{aligned}
\left|I_{2}\right| & \leq \frac{\left|2 \cos ^{2} \theta-\cot ^{2} \theta\right| \cdot \tan \theta}{n \alpha^{2 \rho}}\left(\int_{-\infty}^{0} e^{(2 \rho+1) \alpha x_{2} \tan \theta} d x_{2}+2^{2 \rho} \int_{0}^{\infty} e^{-\alpha x_{2} \tan \theta} d x_{2}\right) \\
& =\frac{\left|2 \sin ^{2}-1\right| \cdot \cos \theta}{n \alpha^{2 \rho} \sin \theta}\left(\frac{1}{(2 \rho+1) \alpha \tan \theta}+\frac{2^{2 \rho}}{\alpha \tan \theta}\right) \\
& =\frac{1}{\alpha^{2 p+1}} \frac{\cos ^{2} \theta \cdot\left|2 \sin ^{2} \theta-1\right|}{\sin ^{2} \theta}\left(\frac{1}{2 \cos ^{2} \theta+1}+2^{2 \cos ^{2} \theta}\right) \\
& \leq \frac{1}{\alpha^{2 p+1}} \frac{\cos ^{2} \theta}{\sin ^{2} \theta}\left(1-\frac{2}{3} \cos ^{2} \theta+1+3 \cos ^{2} \theta\right) \\
& =\frac{1}{\alpha^{2 p+1}} \cdot \frac{72 \cos ^{2} \theta+84 \cos ^{4} \theta}{36 \sin ^{2} \theta} \cdot \frac{1}{n} .
\end{aligned}
$$

Finally, the bounds $|F| \leq 1 / \alpha$ on $\mathbb{R}_{-}$and $|F| \leq 2 / \alpha$ on $\mathbb{R}_{+}$give

$$
\begin{aligned}
\left|I_{3}\right| & \leq \frac{1}{n^{2}}\left(\int_{-2 n}^{-n}+\int_{n}^{2 n} g_{\rho}\left(x_{2}\right)^{2} F\left(x_{2} \tan \theta\right) d x_{2}\right) \\
& \leq \frac{1}{n^{2}}\left(\left(\frac{1}{\alpha}\right)^{2 \rho+1} n+\left(\frac{2}{\alpha}\right)^{2 \rho+1} n\right)=\frac{1}{\alpha^{2 \rho+1}}\left(1+2^{2 \rho+1}\right) \cdot \frac{1}{n} \\
& \leq \frac{1}{\alpha^{2 \rho+1}}(1+2(1+3 \rho)) \cdot \frac{1}{n}=\frac{1}{\alpha^{2 \rho+1}} \cdot\left(3+6 \cos ^{2} \theta\right) \cdot \frac{1}{n} \\
& =\frac{1}{\alpha^{2 \rho+1}} \cdot \frac{108 \sin ^{2} \theta+216 \sin ^{2} \theta \cos ^{2} \theta}{36 \sin ^{2} \theta} \cdot \frac{1}{n} .
\end{aligned}
$$

As a result, we obtain

$$
R\left(h_{n}\right) \leq R\left(g_{\rho}\right)+\left|I_{1}\right|+\left|I_{2}\right|+\left|I_{3}\right| \leq-\left(a-\frac{b}{n}\right)
$$




$$
\begin{aligned}
a & :=-R\left(g_{\rho}\right), \quad b:=\frac{1}{\alpha^{2 \rho+1}} \cdot \frac{B}{36 \sin ^{2} \theta}, \\
B & :=108 \sin ^{2} \theta+72 \cos ^{2} \theta+\left(84 \cos ^{2} \theta+216 \sin ^{2} \theta\right) \cos ^{2} \theta+45 \cos ^{6} \theta-5 \cos ^{8} \theta \\
& =108-36 \cos ^{2} \theta+\left(216-132 \cos ^{2} \theta\right) \cos ^{2} \theta+45 \cos ^{6} \theta-5 \cos ^{8} \theta \\
& =108+180 \cos ^{2} \theta-132 \cos ^{4} \theta+45 \cos ^{6} \theta-5 \cos ^{8} \theta,
\end{aligned}
$$

implying

$$
Q(u)+\frac{\alpha^{2}}{4}\|u\|_{L^{2}(\Omega)}^{2} \leq R\left(h_{n}\right) \leq-\left(a-\frac{b}{n}\right) .
$$

On the other hand,

$$
\begin{aligned}
\|u\|_{L^{2}(\Omega)}^{2} & \leq \int_{-2 n}^{2 n} g_{\rho}\left(x_{2}\right)^{2} F\left(x_{2} \tan \theta\right) d x_{2} \\
& =\int_{-2 n}^{0} F\left(x_{2} \tan \theta\right)^{2 \rho+1} d x_{2}+\int_{0}^{2 n} F\left(x_{2} \tan \theta\right)^{2 \rho+1} d x_{2} \\
& \leq 2 n\left(\frac{1}{\alpha}\right)^{2 \rho+1}+2 n\left(\frac{2}{\alpha}\right)^{2 \rho+1} \leq \frac{1}{\alpha^{2 \rho+1}} \cdot\left(2+4 \cdot 2^{2 \rho}\right) \cdot n \leq c n \\
\text { with } \quad c & :=\frac{6\left(1+2 \cos ^{2} \theta\right)}{\alpha^{2 p+1}},
\end{aligned}
$$

and we have

$$
\mu(\theta, \alpha):=-\frac{\alpha^{2}}{4}-\lambda(\theta, \alpha) \geq \frac{a n-b}{c n^{2}} \quad \text { provided } \quad \text { an }>b .
$$

The right-hand side is optimized by $n=2 b / a$ resulting in $\mu(\theta, \alpha) \geq \frac{a^{2}}{4 b c}=\alpha^{2} \Lambda(\theta)$ with $\Lambda(\theta)$ given in (9).

\section{References}

[1] J. Behrndt, P. Exner, V. Lotoreichik, Schrödinger operators with $\delta$ - and $\delta^{\prime}$-interactions on Lipschitz surfaces and chromatic numbers of associated partitions. Rev. Math. Phys. 26:8 (2014) 1450015.

[2] J. F. Brasche, P. Exner, Yu. A. Kuperin, P. Šeba, Schrödinger operators with singular ineractions. J. Math. Anal. Appl. 184:1 (1994), 112-139.

[3] B. M. Brown, M. S. P. Eastham, I. G. Wood, An example on the discrete spectrum of a star graph. In Analysis on graphs and its applications. (ed. by P. Exner, J. P. Keating, P. Kuchment, T. Sunada, A. Teplyaev), Proc. Symp. Pure Math. 77, Amer. Math. Soc., Providence, RI 2008, 331-335.

[4] B. M. Brown, M. S. P. Eastham, I. G. Wood, Estimates for the lowest eigenvalue of a star graph. J. Math. Anal. Appl. 354:1 (2009), 24-30.

[5] V. Duchêne, N. Raymond, Spectral asymptotics of a broken $\delta$-interaction. J. Phys. A: Math. Theor. 47:15 (2014), 155203.

[6] M. S. P. Eastham, Geometrically induced spectrum of the Schrödinger operator. J. Assoc. Arab Univ. Basic Appl. Sci. 7 (2009), 1-10.

[7] P. Exner, Leaky quantum graphs: a review. In Analysis on graphs and its applications (ed. by P. Exner, J. P. Keating, P. Kuchment, T. Sunada, A. Teplyaev), Proc. Symp. Pure Math. 77, Amer. Math. Soc., Providence, RI 2008, 523-564.

[8] P. Exner, Seize ans après. Appendix K in S. Albeverio, F. Gesztesy, R. Høegh-Krohn, H. Holden, Solvable models in quantum mechanics: Second edition. AMS Chelsea Publ. 350, Amer. Math. Soc., Providence, RI 2005, 453-484.

[9] P. Exner, T. Ichinose, Geometrically induced spectrum in curved leaky wires. J. Phys. A: Math. Gen. 34:7 (2001), 1439-1450.

[10] P. Exner, S. Kondej, Gap asymptotics in a weakly bent leaky quantum wire. J. Phys. A: Math. Theor. 48:49 (2015), 495301.

[11] P. Exner, H. Kovařík, Quantum waveguides. Theor. Math. Phys., Springer, Cham, 2015.

[12] P. Exner, K. Němcová, Bound states in point interaction star graphs. J. Phys. A: Math. Gen. 34:38 (2001), 7783-7794. 
[13] P. Exner, K. Němcová: Leaky quantum graphs: approximations by point interaction Hamiltonians. J. Phys. A: Math. Gen. 36:40 (2003), 10173-10193.

[14] P. Exner, K. Pankrashkin, Strong coupling asymptotics for a singular Schrödinger operator with an interaction supported by an open arc. Comm. Partial Differential Equations 39:2 (2014) 193-212.

[15] P. Exner, S. Vugalter, On the existence of bound states in asymmetric leaky wires. Preprint arXiv:1505.03247

[16] B. Helffer, A. Morame, Magnetic bottles for the Neumann problem: The case of dimension 3. Proc. Indian Acad. Sci. (Math. Sci.) 112:1 (2002), 71-84.

[17] S. Kondej, V. Lotoreichik, Weakly coupled bound state of 2-D Schrödinger operator with potential-measure. J. Math. Anal. Appl. 420:2 (2014), 1416-1438.

[18] V. Lotoreichik, Note on 2D Schrödinger operators with $\delta$-interactions on angles and crossing lines. Nanosyst. Phys. Chem. Math. 4:2 (2013), 166-172.

[19] K. Lu, X.-B. Pan, Surface nucleation of superconductivity in 3-dimensions. J. Differential Equations 168:2 (2000), 386-452.

E-mail address: konstantin.pankrashkin@math.u-psud.fr $U R L:$ http://www.math.u-psud.fr/ pankrash/

Laboratoire de Mathématiques d’Orsay, Univ. Paris-Sud, CNRS, Université, ParisSaClay, 91405 Orsay, France 